\title{
Short-term effects of ambient temperature and relative humidity on the risk of COVID-19 and SARS in Guangzhou, China: A time-series analysis
}

Hui Wang

Guangzhou Centre for Disease Control and Prevention

Chun Chen

Guangzhou Centre for Disease Control and Prevention

Qiaoxuan Lin

Guangzhou Health Information Center, Guangzhou, China

Tiegang Li ( $\triangle$ Huiwang8451@yahoo.com)

Guangzhou Chest Hospital, Guangzhou 510095, China

\section{Research Article}

Keywords: COVID-19; SARS; Meteorological Factors; Distributed lag non-linear model

Posted Date: August 5th, 2020

DOI: https://doi.org/10.21203/rs.3.rs-50445/v1

License: (1) This work is licensed under a Creative Commons Attribution 4.0 International License.

Read Full License 


\section{Abstract}

Coronavirus infection has exerted a severe disease burden on the world, especially the newly emerged SARS-CoV-2 that has caused worldwide pandemic. It is possible meteorological factors can influence the transmission of coronavirus. The aim of this study was to evaluate the effect of meteorological factors on COVID-19 and SARS, and to provide evidence for disease control and prevention. Data of COVID-19 and SARS cases and daily mean temperature, relative humidity and other meteorological factors in Guangzhou in 2003 and 2020 were collected. Using a distributed lag non-linear model approach, we assessed the relationship between ambient temperature, relative humidity and the risks of COVID-19 and SARS. The numbers of cases for COVID-19 and SARS during the study period were 347 and 1072, respectively. There was a dome-shaped relation between mean temperature and COVID-19, with a threshold of $14.50^{\circ} \mathrm{C}(\mathrm{RR}=1.48,95 \% \mathrm{Cl}: 1.01,2.16)$ and the optimal range was $12.40-16.40^{\circ} \mathrm{C}$. A similar association was found between mean temperature and SARS occurrence, with a threshold of $18.40^{\circ} \mathrm{C}$ (RR=1.02, 95\% Cl: $\left.1.00,1.04\right)$, and the optimal range was $15.30-19.30^{\circ} \mathrm{C}$. Besides, there were nonlinear negative relationships between both RH and COVID-19, SARS. In addition, the largest overall effect of RH on COVID-19 and SARS were obtained at $52 \%$ and $45 \%$, yielding relative risk of $7.47(95 \% \mathrm{Cl}: 1.66$, $33.55)$ and 47.56 (95\% Cl: $11.49,196.95)$, respectively. The optimal ranges were below $77.00 \%$ and below $82.70 \%$, respectively. Meteorological parameters should be taken into consideration while developing early warning systems and risk strategies for controlling and preventing coronavirus infection.

\section{Introduction}

In November 2019, a novel coronavirus (SARS-CoV-2) has emerged in Wuhan, and was widespread across mainland China in next few weeks ${ }^{1}$. By the 6th March 2020, a total of 80,151 Coronavirus Disease 2019 (COVID-19) cases have been detected and confirmed in Mainland China. On the 30th January 2020, the World Health Organization (WHO) declared the novel coronavirus "2019-nCoV" outbreak as a global public health emergency. Internationally, as of the 7th May 2020, there were more than 3.5 million additional cases confirmed with 250 thousands deaths reported ${ }^{2}$. Although many coronaviruses are pathogenic to humans but present with mild clinical symptoms, SARS-CoV- 2 is the 2 nd highly pathogenic coronavirus which had severely struck China in the past 2 decades. The first outbreak was SARS firstly emerged in Guangdong province of China. In total, there were 8098 cases of SARS with 774 deaths across 26 countries in 2002-2003 ${ }^{3}$. According to what has been reported, the SARS-CoV-2 shared $79.5 \%$ identity with the SARS-CoV${ }^{4}$. Similar epidemic patterns were observed of the two coronavirus with both the outbreaks occurred during the spring festival. Without vaccines and antiviral medicines, understanding the influential factors for the occurrence and transmission of the SARS-CoV-2 will help deploy contain measures.

Accumulating evidence suggests that meteorological factors, in particularly temperature and humidity might be important environmental factors favoring the transmission of the respiratory infectious disease 5 . For example, a previous study from Hong Kong has shown that during the epidemic, the risk of 
increased daily incidence of SARS was 18.18-fold higher in days with a lower air temperature than in days with a higher temperature ${ }^{6}$. Liu ${ }^{7}$ and Zhang ${ }^{8}$ explored the associations of temperature with H7N9 human cases. They both reported that temperature contributed significantly to human infection with $\mathrm{H} 7 \mathrm{~N}$ 9, and the effects of temperatures were nonlinear. Studies from temperate and subtropical climates have demonstrated that low temperature and humidity increased the risk of influenza ${ }^{9,10}$. It is plausible SARS-CoV-2 can remain viable and infectious in aerosol for multiple hours and on surfaces up to days ${ }^{11}$, and some experimental studies as well as animal models have shown that low specific humidity favored virus survival and aerosol transmission ${ }^{12-14}$. Moreover, Salah et al. revealed that breathing cold air would slow mucociliary clearance and thereby encouraged viral spread within the respiratory tract ${ }^{15}$.

Despite the research advance, only a few studies have explored environmental parameters of coronavirus infection. The association between temperature, humidity and the occurrence of coronavirus has remained unclear. As Guangzhou and Wuhan sharing similar climate patterns were both severely attacked by the SASR and COVID-19, are there any environmental factors facilitating the COVID-19 and SARS outbreak in China? The objective of the present study was to examine the relations between temperature, humidity and the risk of coronavirus infections in a subtropical climatic zone, Guangzhou. We collected the COVID-19 and SARS cases information reported in 2020 and 2003 in Guangzhou. Our hypotheses were that there were significant associations between mean temperatures (MT), relative humidly $(\mathrm{RH})$ and coronavirus infection and the relationships were non-linear.

\section{Results}

\section{Basic information in SARS and COVID-19}

Between Jan 2nd and May 11th 2003, a total of 1072 SARS cases aged 2 month to 92 years were reported. Of the 1072 cases, 475 were male and 597 were female, with a male-to-female sex ratio1:1.26. There was an average of 7.68 daily SARS cases, and the mean level of daily MT and RH were $18.77^{\circ} \mathrm{C}$ and $76.95 \%$ at the same time. COVID-19 cases were first reported at Jan 21st 2020, the date was very close to the first SARS case in Guangzhou 17 years ago. Between Jan 21st and Mar 6th 2020, a total of 347 COVID-19 cases aged 3 months to 90 years were reported. Of the 347 cases, 167 were male and 180 were female, with a male-to-female sex ratio 1:1.08. There was an average of 9.35 daily COVID-19 cases and the mean level of daily MT and $\mathrm{RH}$ were $15.69^{\circ} \mathrm{C}$ and $78.78 \%$, respectively (Table1). Fig. 1 shows the trends of reported cases and meteorological variables during the period of study. Visually, there was a similar trend observed in the association between COVID-19 cases, SARS cases and meteorological factors.

\section{Estimation of Separate effects of MT, RH on COVID-19 and SARS}

Fig. 2 shows the three-dimensional relationship between MT, RH and COVID-19 as well as SARS cases within 5 lag days, taking the median values as the reference. The largest separate effects of MT on COVID-19 and SARS were obtained at $13.70^{\circ} \mathrm{C}$ and $11.10^{\circ} \mathrm{C}$, although the effects were not significant. 
And the largest separate effects of RH on COVID-19 and SARS were obtained at $52.00 \%$ and $45.00 \%$, yielding relative risk of $2.28(95 \% \mathrm{Cl}: 1.30,3.98)$ and $3.73(95 \% \mathrm{Cl}: 2.38,5.85)$ respectively.(Seen in Table 2$)$

Estimation of Overall effects of MT, RH on COVID-19 and SARS

Fig. 3 shows the overall effects of MT and RH within 5 lag days, taking the median values as references. The overall cumulative association analyses suggested non-linear relationship between MT, RH and COVID-19 as well as SARS. There was a dome-shaped relation between MT and COVID-19, with a threshold of $14.50^{\circ} \mathrm{C}(\mathrm{RR}=1.48 \times 95 \% \mathrm{Cl}: 1.01,2.16)$ and the optimal range was $12.40-16.40^{\circ} \mathrm{C}$. There was a similar association between MT and SARS occurrence, with a threshold of $18.40^{\circ} \mathrm{C}(\mathrm{RR}=1.02,95 \% \mathrm{Cl}: 1.00$, 1.04), and the optimal range was $15.30-19.30^{\circ} \mathrm{C}$. Besides, there were negative relationships between COVID-19, SARS and RH. The largest overall effect of RH on COVID-19 and SARS were obtained at $52.00 \%$ and $45.00 \%$, yielding relative risk of 7.47 (95\% Cl: $1.66,33.55)$ and $47.56(95 \% \mathrm{Cl}: 11.49,196.95)$, respectively. The optimal ranges were below $77.00 \%$ and below $82.70 \%$, respectively.

\section{Discussion}

Coronavirus infection has become global public health threat that causes severe respiratory disease with a high case fatality ${ }^{17}$. In this study, we analyzed the relationships between the epidemics of COVID-19, SARS and MT as well as RH using the DLNM. Results suggested that COVID-19 and SARS exhibited similar trend of RR curves with the largest overall effects at similar $\mathrm{MT}\left(14.5^{\circ} \mathrm{C}, 18.4^{\circ} \mathrm{C}\right.$ respectively) and $\mathrm{RH}(52 \%, 45 \%)$ in Guangzhou.

The overall effects of MT on COVID-19 as well as SARS exhibited dome -shaped curves. Specifically, the risks of COVID-19 and SARS rose along with MT up to certain points with largest effects and then subsequently decreased with increasing MT. The finding was similar to a previous study on SARS that ambient temperate was negatively associated with SASR outbreaks in Hong Kong ${ }^{18}$. Whereas, our results were less consistent with a recent published study on COVID-19. By collecting the cumulative number of confirmed cases from 224 cities in China, Yan et al. found that ambient temperature has no significant impact on the transmission ability of SARS-CoV-2. These discrepancies can be explained by different modeling approaches, controlling efforts and also medical resource. In addition, the response to meteorological factors of coronavirus infection may be varied with the different climate environments 18,19. Thus, more studies from different climate zones are needed to expand our knowledge of this relationship. What is worth noticing, our study, for the first time found that $\mathrm{RH}$ was negatively correlated with the occurrence of COVID-19 and SARS. Our results were also similar to previous reports where MERSCoV cases increased with lower MT and RH. For instance, Gardner et al found that lowest temperature $(\mathrm{OR}=1.27 ; 95 \% \mathrm{Cl}: 1.04-1.56)$ and humidity $(\mathrm{OR}=1.35 ; 95 \% \mathrm{Cl}: 1.10-1.65)$ were associated with increased risk of MERS-CoV infection ${ }^{20}$. These findings combined with previous studies suggested that cold and dry weather conditions may facilitate an optimal environment for the occurrence and transmission of respiratory infectious disease caused by coronavirus. 
Temperature and relative humidity have been shown to exert significance on causing viral respiratory infectious disease ${ }^{21,22}$. Jaakkola has conducted a cross-over study in a cold climate ${ }^{23}$. They found that every $1^{\circ} \mathrm{C}$ decrease in temperature and $0.5 \mathrm{~g}$ decrease per $\mathrm{m}^{3}$ in absolute humidity increased the estimated risk by $11 \%$ and $58 \%$, respectively. Soebiyanto et al. have reported that influenza activity was negatively associated to temperature ${ }^{24,25}$. This kind of associations were also observed in H5N1 and H7N9 with the highest frequency in a temperature range of $5-10^{\circ} \mathrm{C}$ and $10-15^{\circ} \mathrm{C}$, respectively ${ }^{26}$. Multiple mechanisms may contribute to the relationships between the meteorological factors and coronavirus infection. It has been reported that the coronavirus can survive in the environment, and be transmitted through close contacts ${ }^{27}$. A laboratory study has reported infectious virus persisted for as long as 28 days at $4^{\circ} \mathrm{C}$, and the lowest level of inactivation occurred at $20 \% \mathrm{RH}^{28}$. Chan et al. observed that virus viability was rapidly lost at higher temperatures and higher relative humidity (e.g., 38॰C, and relative humidity $>95 \%{ }^{29}$. Additionally, Chin demonstrated that low temperature enhanced the survivability of viruses and provides more suitable conditions for transmission ${ }^{30}$. Other explanatory factors including alterations in host susceptibility (e.g. immunity), viral mutations, as well as the effects of weather on social contact patterns during the wintertime can also partly explained the association between MT, RH and coronavirus infection. For instance, in low-humidity environments, immune defense system is impaired and the risk of infectious diseases rises ${ }^{31}$.

There were several limitations in our study. First, in the COVID-19 and SARS model, a limited timespan for dataset in our study might influence the relationships between the occurrence of coronavirus infection and meteorological factors. Second, we only adjusted the concomitant variable of meteorological factors in the study. Other factors such as host susceptibility, prevention and control efforts might have effects on the associations between meteorological factors and coronavirus infection. All of these will be considered in a more comprehensive model in the future. Given the gap in knowledge, our results provided a good starting point and a priori hypothesis for further studies.

In conclusion, our study suggests that both low MT and RH were associated with the occurrence of coronavirus infection during wintertime in subtropical climate. The finding may partly clarify the reason that the SARS-CoV and SARS-CoV-2 caused extensive outbreak in southern China where the climate were characterized as subtropical climate and the MT and RH reached the lowest between November to February in the next year. And also our research highlights that governments should take meteorological parameters into consideration while developing early warning systems and risk strategies for controlling and preventing coronavirus infection. Further studies are still needed to explain the relationships and confirm causality between temperature, humidity and coronavirus infection.

\section{Methods}

Study location 
Guangzhou偖 the capital of Guangdong Provinces located in Southern China $\left(112^{\circ} 57 \mathrm{E}\right.$ to $114^{\circ} 3 \mathrm{E}$ and $22^{\circ} 26 \mathrm{~N}$ to $23^{\circ} 56 \mathrm{~N}$ ) which is the third largest city of China, with a permanent resident population of approximately 14.90 million at the end of 2019 . It has a humid subtropical climate with comparatively longer summers and shorter winters (Lu et al., 2009).

Study design and Data collection

A retrospective study to investigate the relationship between COVID-19, SARS cases and meteorological factors were carried out. Daily count of reported COVID-19 cases in Guangzhou from 21st Jan 2020 to 8th Mar 2020 was obtained from the China Information System for Disease Control and Prevention (CISDCP). And the daily count of reported SARS cases in Guangzhou from 2nd Jan 2003to 11th May 2003was obtained from the archives of SARS in Guangzhou center for disease control and prevention. In this study, all the COVID-19 and SARS cases were all laboratory confirmed. Daily meteorological factors including MT, RH and wind velocity (WV) corresponding to the case report in 2003 and 2020 were obtained from Guangzhou meteorological bureau.

Statistical analysis

Mean \pm standard deviation $(x \pm s)$ and percentile were used to describe the distribution of cases and meteorological factors. As daily count of COVID-19 and SARS cases typically follow a quasi-Poisson distribution, a quasi-Poisson generalized linear regression combined with a distributed lag non-linear model (DLNM) was applied to quantify the effect of MT and RH on COVID-19 and SARS. In this study, we found the effect of MT and RH was negligible for lags above 5 days, so a maximum lag of 5 days was used to explore the lag associations. To adjust for potential effects of meteorological conditions, we used a smooth function of the natural cubic spline in the DLNM for wind velocity. In all cases, the Minimum Akaike Information Criterion (AIC) was adopted to choose the degree of freedom (df) for meteorological factors, lag and time trend. The model was specified as:

$\log \left[E\left(Y_{t}\right)\right]=a+c b($ temperature/relative humidity) + ns(humidity_lag2/temperature_lag2,df)+ns(wind velocity_lag2, df)+ ns(time, df)

In the model, $Y t$ is reported daily COVID-19 /SARS cases on day $t ; a$ is the intercept; $c b(M T / R H)$ indicates the cross-basis function, which is obtained by DLNM to model non-linear and distributed lag effects of temperature/humidity. The "ns" means a smooth function based on natural cubic spline. Humidity_lag2 /temperature_lag2 /wind velocity_lag2 are humidity, temperature and wind velocity with two-day lag .Time controls the time trend. All the analyses were carried out using the "dlnm" and "spline" packages in $R$ software (version 3.6.2).

\section{Declarations}

Acknowledgments 
We would give our special thanks to CISDCP and Guangzhou meteorological bureau for providing the data.

\section{Author contributions}

TG Li designed the study. H Wang and C Chen performed data analysis, and wrote the study. QX Lin contributed to the data and performed data analysis collection. All authors assisted in writing the manuscript.

\section{Funding}

This work was supported The Project for Key Medicine Discipline Construction of Guangzhou Municipality (grant number 2017-2019-07)

Conflict of interest

The authors have no other conflicts of interest or funding to disclose

\section{References}

$1 \quad \mathrm{Li}, \mathrm{Q}$. et al., Early Transmission Dynamics in Wuhan, China, of Novel Coronavirus-Infected Pneumonia. N Engl J Med (2020).

2 World Health Organization, Coronavirus disease (COVID-2019) situation reports. https://www.who.int/docs/default-source/coronaviruse/situation-reports/20200507covid-19-sitrep108.pdf?sfvrsn=44cc8ed8_2. 2020-05-07.

$3 \quad$ World Health Organization, Summary of probable SARS cases with onset of illness from 1 November 2002 to 31 July 2003. Yang, Y., Peng, F., Wang, R., Guan, K., Jiang, T., Xu, G., Sun, J., Chang, C., 2020. The deadly coronaviruses: The 2003 SARS pandemic and the 2020 novel coronavirus epidemic in China. JOURNAL OF AUTOIMMUNITY 109, 102434. http://doi.org/10.1016/j.jaut.2020.102434.

4 Zhou, P. et al., A pneumonia outbreak associated with a new coronavirus of probable bat origin. NATURE 579270 (2020).

5 Tan, J. et al., An initial investigation of the association between the SARS outbreak and weather: with the view of the environmental temperature and its variation. J Epidemiol Community Health 59186 (2005).

6 Lin, K., Yee-Tak, F. D., Zhu, B. \& Karlberg, J., Environmental factors on the SARS epidemic: air temperature, passage of time and multiplicative effect of hospital infection. EPIDEMIOL INFECT 134223 (2006). 
Liu, T. et al., Independent and interactive effects of ambient temperature and absolute humidity on the risks of avian influenza $A(H 7 N 9)$ infection in China. SCI TOTAL ENVIRON619-620 1358 (2018).

$8 \quad$ Zhang, Y., Cindy, F., Ma, C., Yang, P. \& Wang, Q., The impact of temperature and humidity measures on influenza A (H7N9) outbreaks-evidence from China. INT J INFECT DIS(2014).

9 Dai, Q. et al., The effect of ambient temperature on the activity of influenza and influenza like illness in Jiangsu Province, China. SCI TOTAL ENVIRON645 684 (2018).

10 Meng, H. et al., Healthcare seeking behavior of patients with influenza like illness: comparison of the summer and winter influenza epidemics. BMC INFECT DIS 16499 (2016).

van Doremalen, N. et al., Aerosol and surface stability of HCoV-19 (SARS-CoV-2) compared to SARS-CoV-1. medRxiv 2020 (2020).

Lowen, A. C., Mubareka, S., Steel, J. \& Palese, P., Influenza virus transmission is dependent on relative humidity and temperature. PLOS PATHOG 31470 (2007).

Shaman, J. \& Kohn, M., Absolute humidity modulates influenza survival, transmission, and seasonality. Proc Natl Acad Sci U S A 1063243 (2009).

14 Ssematimba, A., Hagenaars, T. J. \& de Jong, M. C., Modelling the wind-borne spread of highly pathogenic avian influenza virus between farms. PLOS ONE 7 e31114 (2012).

15 Salah, B., Dinh, X., Fouilladiu, J. L., Lockhart, A. \& Regnard, J., Nasal mucociliary transport in health subjects is slower when breathing dry air. EUR RESPIR J 1846 (1988).

16 Lu, L. et al., Time series analysis of dengue fever and weather in Guangzhou, China. BMC PUBLIC HEALTH9 395 (2009).

17 Yang, Y. et al., The deadly coronaviruses: The 2003 SARS pandemic and the 2020 novel coronavirus epidemic in China. J AUTOIMMUN109 102434 (2020).

18 Tan, J. et al., An initial investigation of the association between the SARS outbreak and weather: with the view of the environmental temperature and its variation. J Epidemiol Community Health 59186 (2005).

Lofgren, E., Fefferman, N. H., Naumov, Y. N., Gorski, J. \& Naumova, E. N., Influenza seasonality: underlying causes and modeling theories. J VIROL 815429 (2007).

Gardner, E. G. et al., A case-crossover analysis of the impact of weather on primary cases of Middle East respiratory syndrome. BMC INFECT DIS 19113 (2019). 

Years of County-Level Evidence from the United States. AM J EPIDEMIOL 176 S114 (2012).

22 Tamerius, J. et al., Global Influenza Seasonality: Reconciling Patterns across Temperate and Tropical Regions. ENVIRON HEALTH PERSP119 439.

23 Jaakkola, K. et al., Decline in temperature and humidity increases the occurrence of influenza in cold climate. ENVIRON HEALTH-GLOB 1322.

24 Soebiyanto, R. P. et al., The role of temperature and humidity on seasonal influenza in tropical areas: Guatemala, El Salvador and Panama, 2008-2013. PLOS ONE 9 e100659 (2014).

Soebiyanto, R. P. et al., Associations between Meteorological Parameters and Influenza Activity in Berlin (Germany), Ljubljana (Slovenia), Castile and Leon (Spain) and Israeli Districts. PLOS ONE10 e134701 (2015).

26 Liu, T. et al., Independent and interactive effects of ambient temperature and absolute humidity on the risks of avian influenza $A(H 7 N 9)$ infection in China. SCI TOTAL ENVIRON619-620 1358 (2018).

27 Casanova, L. M., Jeon, S., Rutala, W. A., Weber, D. J. \& Sobsey, M. D., Effects of air temperature and relative humidity on coronavirus survival on surfaces. Appl Environ Microbiol 762712 (2010).

28 Casanova, L. M., Jeon, S., Rutala, W. A., Weber, D. J. \& Sobsey, M. D., Effects of air temperature and relative humidity on coronavirus survival on surfaces. Appl Environ Microbiol 762712 (2010).

29 Chan, K. H. et al., The Effects of Temperature and Relative Humidity on the Viability of the SARS Coronavirus. Advances in Virology (2011).

30 Chin, A. et al., Stability of SARS-CoV-2 in different environmental conditions. medRxiv 2020 (2020).

31 Kudo, E. et al., Low ambient humidity impairs barrier function and innate resistance against influenza infection. Proceedings of the National Academy of Sciences 11610905 (2019).

\section{Tables}

Table 1. Descriptive statistics for daily COVID-19, SARS cases and MT, RH variables in Guangzhou 2003 and 2020 


\begin{tabular}{llllllllll}
\hline & Year & Variable & Mean & SD & Min & P25 & Median & P75 & Max \\
\hline COVID-19 & 2020 & Cases & 9.35 & 10.73 & 0.00 & 1.00 & 4.00 & 16.00 & 38.00 \\
& & MT & 15.69 & 3.89 & 9.40 & 11.82 & 16.40 & 18.80 & 21.70 \\
& & RH & $\mathbf{7 8 . 7 8}$ & $\mathbf{1 1 . 4 8}$ & $\mathbf{5 2 . 0 0}$ & $\mathbf{7 2 . 7 5}$ & $\mathbf{8 0 . 5 0}$ & $\mathbf{8 6 . 5}$ & $\mathbf{9 8 . 0 0}$ \\
SARS & 2003 & Cases & $\mathbf{7 . 6 8}$ & $\mathbf{9 . 1 8}$ & $\mathbf{0 . 0 0}$ & 2.00 & $\mathbf{4 . 0 0}$ & $\mathbf{9 . 5 0}$ & $\mathbf{4 1 . 0 0}$ \\
& & MT & 18.77 & 4.48 & 11.10 & 15.15 & 19.30 & 22.25 & 26.80 \\
& & RH & $\mathbf{7 6 . 9 5}$ & 10.59 & $\mathbf{4 5 . 0 0}$ & $\mathbf{7 2 . 0 0}$ & $\mathbf{7 8 . 0 0}$ & $\mathbf{8 4 . 0 0}$ & $\mathbf{9 7 . 0 0}$ \\
\hline
\end{tabular}

SD: Standard Deviation; Cases: daily reported cases; MT: daily mean temperature; RH: daily relative humidity

Table 2. The largest separate and overall effects of meteorological factors values of COVID-19 and SARS in Guangzhou

\begin{tabular}{|c|c|c|c|c|c|c|c|}
\hline \multirow{2}{*}{\multicolumn{2}{|c|}{ iable }} & \multirow{2}{*}{$\begin{array}{l}\text { Reference } \\
\text { value }\end{array}$} & \multicolumn{3}{|c|}{ Separate effect } & \multicolumn{2}{|c|}{ Overall effect } \\
\hline & & & $\begin{array}{l}\text { Maximum RR } \\
(95 \% \mathrm{CI})\end{array}$ & $\begin{array}{c}\text { Variable } \\
\text { value }\end{array}$ & lag & Maximum RR & $\begin{array}{c}\text { Variable } \\
\text { value }\end{array}$ \\
\hline \multirow[t]{4}{*}{ )- } & MT & $16.40^{\circ} \mathrm{C}$ & $1.08(0.90,1.30)$ & $13.70^{\circ} \mathrm{C}$ & 0 & $1.48(1.01,2.16)$ & $14.50^{\circ} \mathrm{C}$ \\
\hline & RH & $80.50 \%$ & $2.28(1.30,3.98)$ & $52.00 \%$ & 0 & $7.47(1.66,33.55)$ & $52.00 \%$ \\
\hline & MT & $19.30^{\circ} \mathrm{C}$ & $1.03(0.93,1.14)$ & $11.10^{\circ} \mathrm{C}$ & 3 & $1.02(1.00,1.04)$ & $18.40^{\circ} \mathrm{C}$ \\
\hline & RH & $78.00 \%$ & $3.73(2.38,5.85)$ & $45.00 \%$ & 3 & $\begin{array}{c}47.56 \\
(11.49,196.95)\end{array}$ & $45.00 \%$ \\
\hline
\end{tabular}

MT: daily mean temperature; RH: daily relative humidity

\section{Figures}
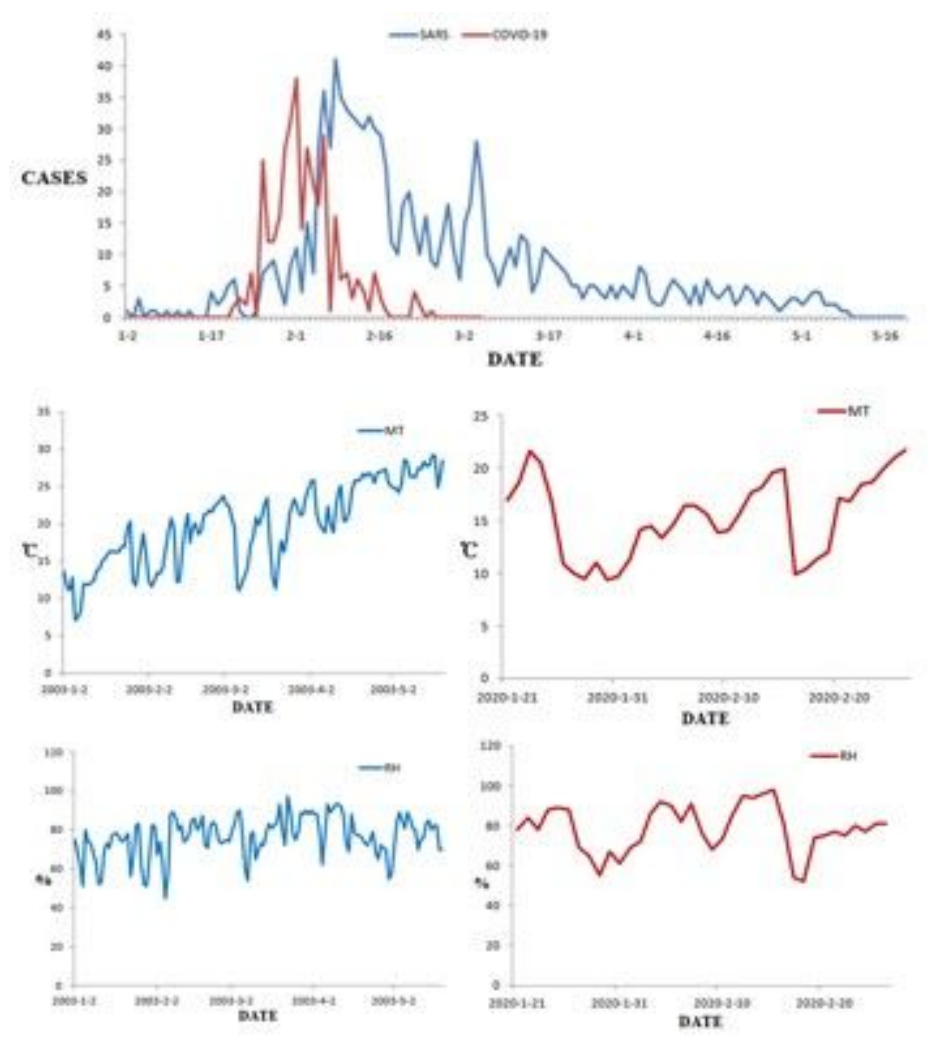
Figure 1

Time series distribution of daily COVID-19 case count, SARS cases count and meteorological variables during the study period.

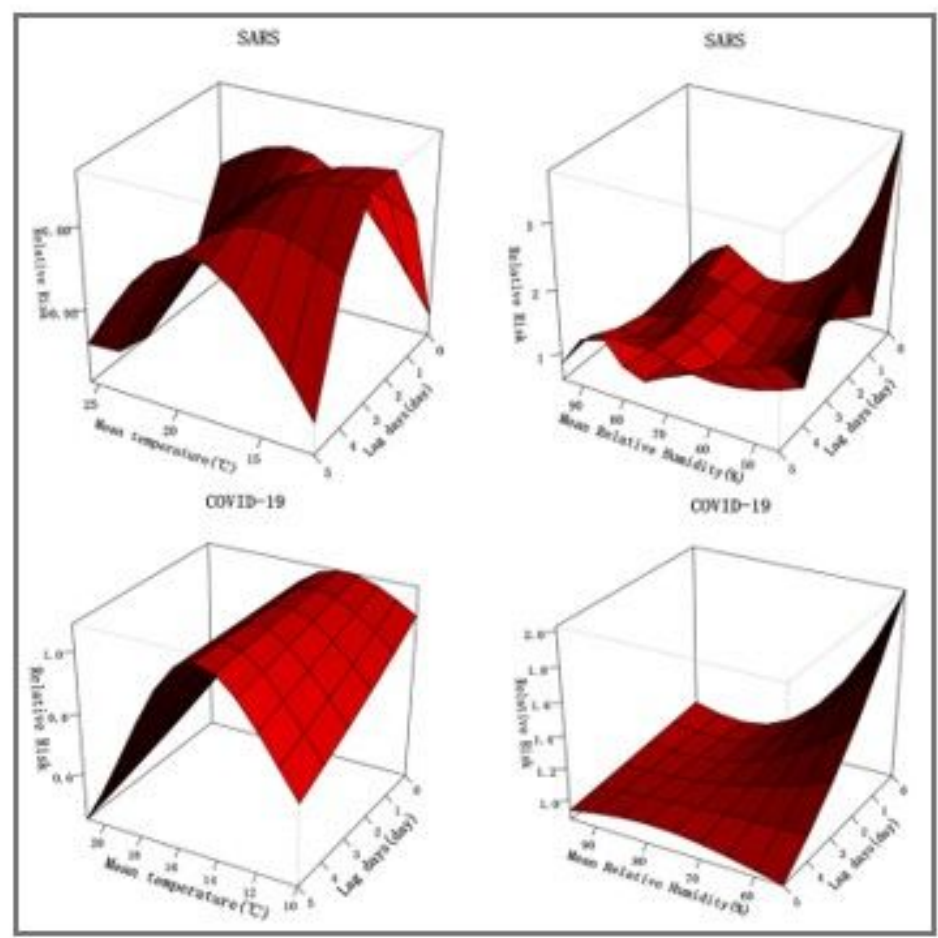

Figure 2

3D plots of relative risk of MT and RH on COVID-19 and SARS within 5 lag days 

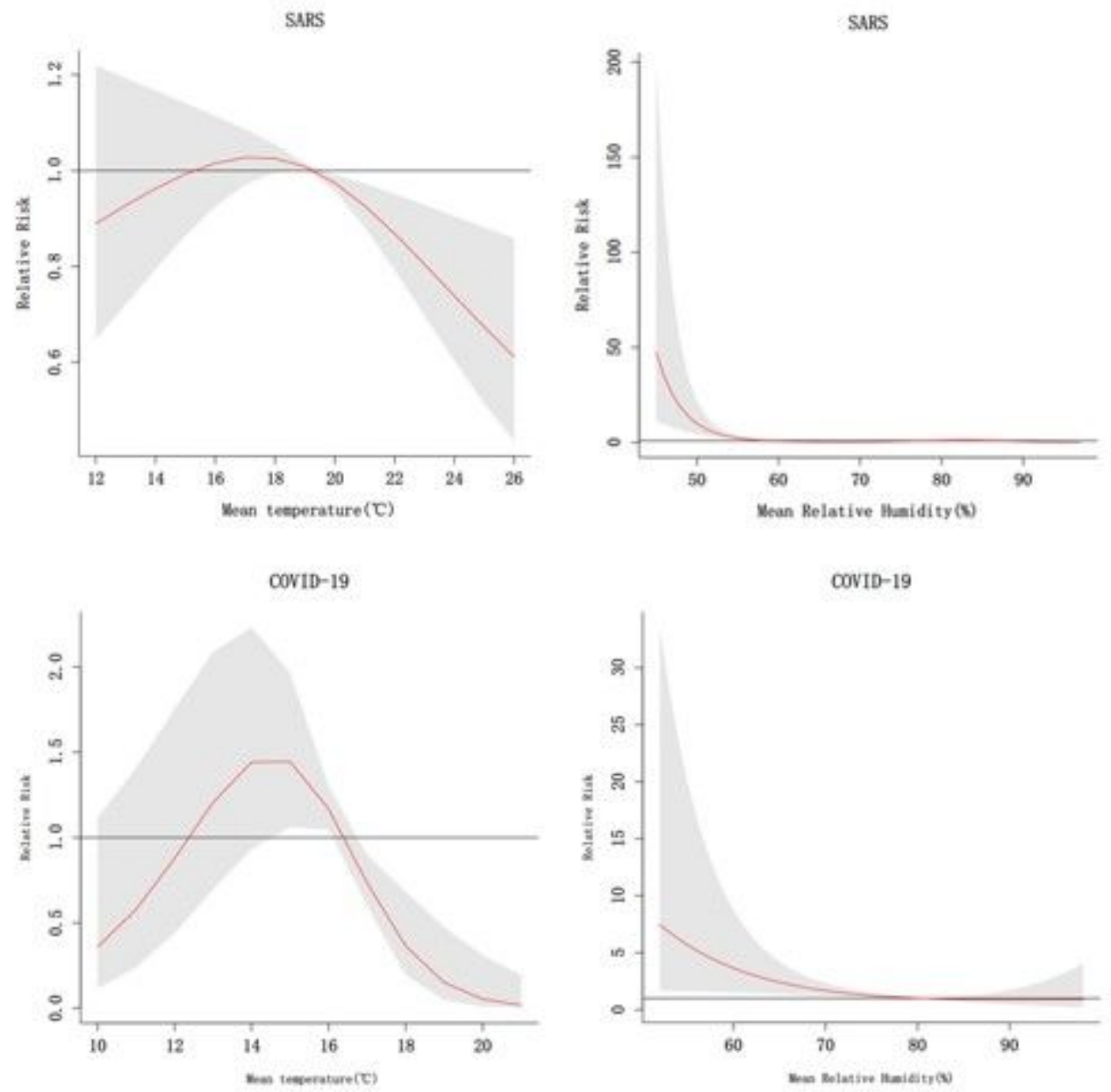

Figure 3

Overall effects of MT and RH on COVID-19 and SARS within 5 lag days. The median values of meteorological factors was selected as references 